\title{
Interactive Visual Analysis and Exploration of Injection Systems Simulations
}

\author{
Krešimir Matković* \\ VRVis Research Center Vienna
}

\author{
Mario Jelović ${ }^{\dagger}$ \\ AVL-AST Zagreb, Croatia
}

\author{
Josip Jurić, Zoltan Konyha \\ VRVis Research Center Vienna
}

\author{
Denis Gračanin§ \\ Virginia Tech
}
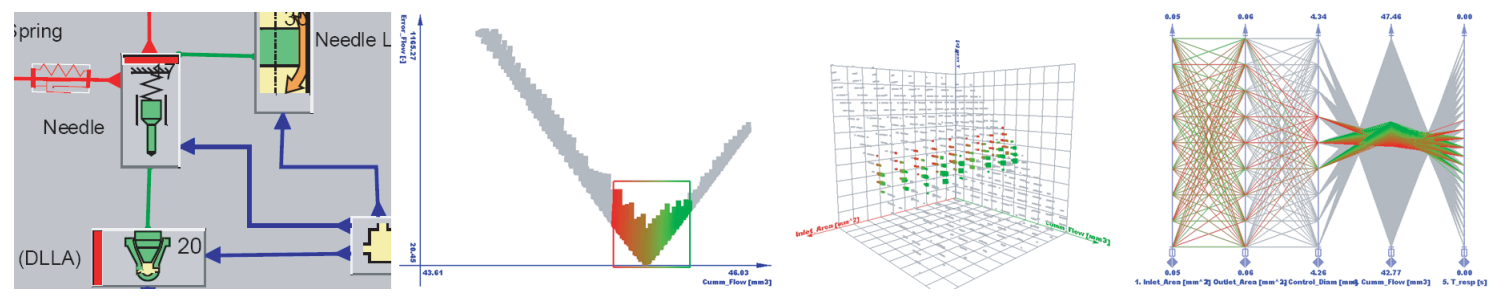

Figure 1: Injection systems: from simulation to visualization and exploration.

\begin{abstract}
Simulations often generate large amounts of data that require use of SciVis techniques for effective exploration of simulation results. In some cases, like 1D theory of fluid dynamics, conventional SciVis techniques are not very useful. One such example is a simulation of injection systems that is becoming more and more important due to an increasingly restrictive emission regulations. There are many parameters and correlations among them that influence the simulation results. We describe how basic information visualization techniques can help in visualizing, understanding and analyzing this kind of data. The ComVis tool is developed and used to analyze and explore the data. ComVis supports multiple linked views and common information visualization displays such as $2 \mathrm{D}$ and 3D scatter-plot, histogram, parallel coordinates, pie-chart, etc. A Diesel Common Rail Injector with $2 / 2$ Way Valve is used for a case study. Data sets were generated using a commercially available AVL HYDSIM simulation tool for dynamic analysis of hydraulic and hydro-mechanical systems, with the main application area in the simulation of fuel injection systems.
\end{abstract}

CR Categories: J.2.5 [Computer Applications]: PHYSICAL SCIENCES AND ENGINEERING-Engineering; I.3.8 [Computing Methodologies]: COMPUTER GRAPHICS—Applications I.3.0 [Computing Methodologies]: COMPUTER GRAPHICSGeneral

Keywords: Information visualization, visual exploration, simulation, injection system

\section{INTRODUCTION}

Simulation plays an important role in the modern automotive engine design. Requests for shorter time to market and constantly increasing engine complexity make the use of simulation in a design process a must. Simulation of the injection systems is only a part of the whole engine simulation chain. It is especially important when engine designers want to meet current very demanding

\footnotetext{
*e-mail: Matkovic@VRVis.at

$\dagger$ e-mail:mario.jelovic@avl.com

†e-mail:Juric,Konyha@VRVis.at

§e-mail:gracanin@vt.edu
}

emission criteria. For this paper we have used the AVL HYDSIM simulation tool [1]. It is based on the 1D theory of fluid dynamics. This means that geometry is taken into account, but it is approximated with 1D models. A pipe, e.g. is considered to be a line. If the pressure in a pipe has to be computed, the result will be a function of one parameter (axial position). The pressure is considered to be constant on the slice perpendicular to the pipe axis.

The simulation output data is often visualized using scientific visualization (SciVis) methods [17]. Although SciVis methods could have been applied to the injection simulation data, the lack of real 3D spatial information makes the data more convenient for information visualization (InfoVis) techniques. For the visualization, exploration, and analysis the engineers are currently using a set of static 2D charts and numerical tables. For optimization of the model pure numerical methods are often used [10]. As a valuable addition to numerical optimization methods, we propose the use of interactive InfoVis techniques for exploration, analysis and getting insight into the injection simulation data $[18,19]$. Our intention is not to replace the numerical optimization methods, but to assist engineers in understanding the simulation results and how they are influenced by the model parameter changes. This is not only helpful for novice engineers, but also for experienced engineers when they are confronted with a complex design. All information visualization principles and methods described in this paper are implemented in the ComVis software tool [5, 20].

The remainder of the paper is organized as follows. Section 2 describes characteristics of the Diesel common rail injection system used for the case study. Section 3 discusses the use of basic views and interaction techniques for information visualization. Section 4 illustrates analysis and exploration of simulation results while Section 5 concludes the paper.

\section{INJECTION SIMULATION}

Modern emission regulations are one of the driving forces in designing and improving injection systems today. The AVL hydraulic simulation software tool HYDSIM [1] is a modular program for the dynamic analysis of hydraulic and hydro-mechanical systems. It is based on the theory of fluid dynamics (1D) and vibration of multi-body systems (2D). The main application area of HYDSIM is simulation of fuel injection systems. The user defines a model using 2D graph-like structures with icons and connecting elements. The defined HYDSIM model provides a general representation of the system topology. Figure 2 shows a snapshot of the application window. For each element (represented by an icon) the user can 


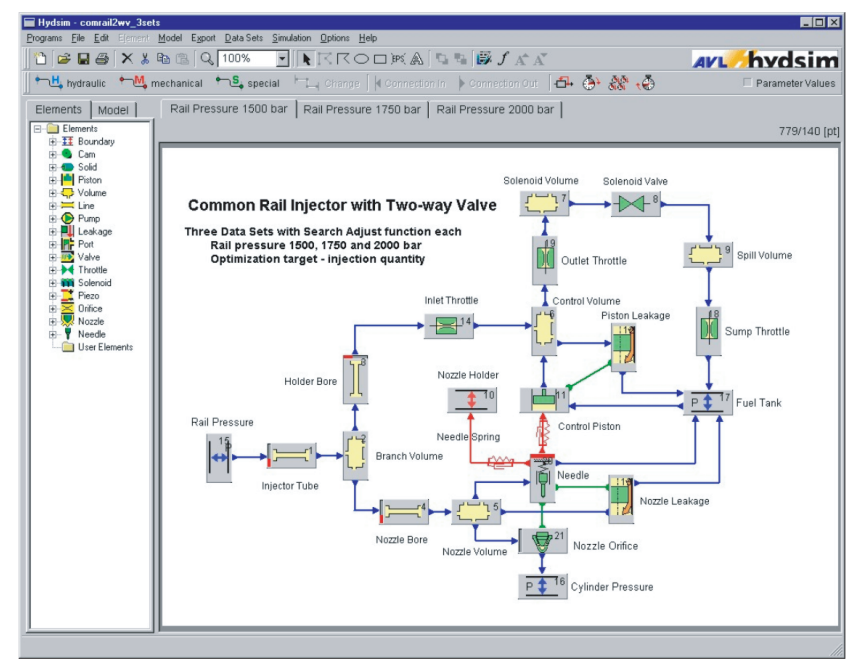

Figure 2: A snapshot of the AVL HYDSIM simulation tool. The model is built as a 2D graph-like structure with elements and connecting lines, each with a set of parameters.

specify properties for the particular case. Once a definition of the model is completed, the simulation provides output parameters. In a typical workflow, engineers analyze these results and, if necessary, modify the model, and repeat the simulation until the desired results are achieved.

\subsection{Diesel Common Rail Injection System}

A special type of injection systems - the common rail injection is used as a case study. The common rail injection system has been identified as an attractive injection system for Diesel car engines [3]. It is offered by all major car manufacturers today. It operates at very high pressure level using a flexible electronic control of the fuel delivery, injection timing, injection pressure and rate of injection by multiple injection strategy. By controlling these parameters, the common rail is capable of achieving a level of performance and driving comfort for Diesel cars similar to that of gasoline powered models with less fuel consumption and low exhaust emissions $[8,9]$.

The common rail injection system is the most flexible injection system for design and application of diesel injection system. The most important requirements for an injection system include:

- high injection pressure that can be chosen independently,

- high accuracy of injected fuel quantity,

- flexible injection timing control,

- capability of flexible pilot injection, and

- capability of small injection quantities.

Engine manufacturers are responding to those requirements by providing increasingly sophisticated and complex technical solutions.

\subsection{Injector Design and Injection Process}

The injector is the central part of an injection system that injects a desired fuel quantity into the cylinder. Figure 3 shows a typical injector with main components. The common rail injector system is controlled by a $2 / 2$ solenoid operated valve. The main components of the injector include:

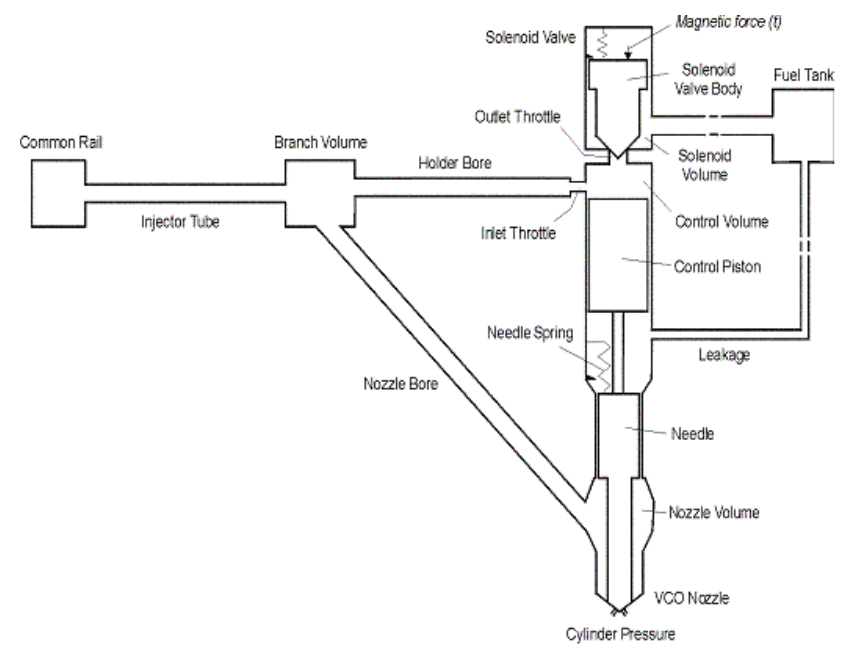

Figure 3: Main components of a common rail injector system.

- needle with the control piston,

- multi hole VCO nozzle,

- two orifices controlling the pressure in the control chamber and a corresponding control piston (inlet and outlet throttle), and

- solenoid valve body with opening/closing throttle.

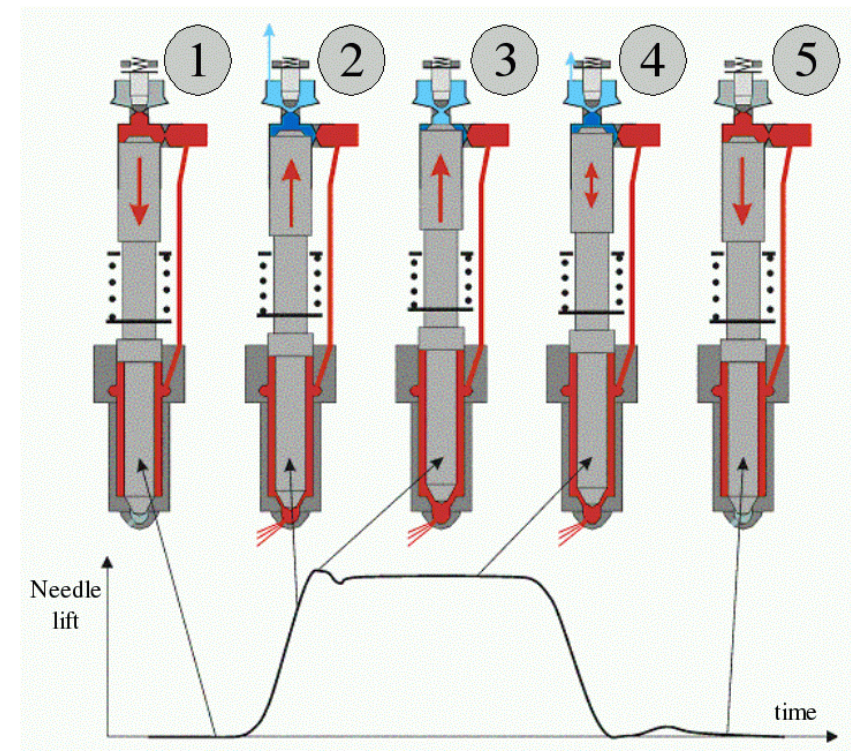

Figure 4: Common rail injector operation: 1. ECU activates the solenoid valve. 2. The outlet throttle opens. Fuel flow causes a pressure drop in the control chamber. Low pressure results in the needle going up. Injection starts. 3. The needle is open. 4. Injection has the best performances. ECU starts releasing the solenoid valve. 5. The outlet throttle closes. Pressure in the control chamber and the spring force move the needle down. Injection stops.

The principle of the common rail injector operation is outlined in Figure 4 . The red, blue and sky blue colors present pressure levels. 
The injection process is initiated by energizing the solenoid valve by the ECU (Electronic Control Unit) (Figure 4.1). As a result, the outlet throttle opens. Fuel flows through the control volume towards the solenoid volume, and as a result the pressure in the control volume drops. The pressure difference between the top and bottom sides of the needle pushes the needle up (Figure 4.2), at the same time opening the injection nozzle. After some time the needle reaches the stable open position resulting in the optimum injection of fuel into the cylinder (Figure 4.3). When the solenoid valve is deactivated by the ECU, the spring in the solenoid valve closes the outlet throttle in the injector (Figure 4.4). As a result, the pressure in the control volume increases. The pressure on the top side of the control piston, together with the nozzle spring force, closes the nozzle and injection process ends (Figure 4.5).

The injected fuel quantity depends mainly on the length of the time interval the nozzle was open, the nozzle geometry and the injection pressure, which in turn depends on many other model parameters. The most dominant model parameters include inlet and outlet throttle geometries, common rail pressure, and solenoid valve dynamics. The HYDSIM tool allows for a simulation of multiple cases where all those parameters can change. That results in a large number of simulation output data sets. Understanding the influence of parameter variations on the injection simulation results is a challenging task. information visualization techniques can be used to assist engineers in that task, as we are going to show in the following sections.

\section{USING INFOVIS TECHNIQUES WITH INJECTION SIMULA- TION DATA}

Plain data tables or simple 2D charts are not very useful when applied to large data sets. Furthermore, users often look for various correlations and want to compare specific data sets. Information visualization attempts to make it easier for a user to analyze and explore large data sets by cleverly and interactively displaying information [4, 2]. We use five basic InfoVis views to assist engineers in analyzing and understanding the injection simulation results.

\subsection{Basic Views}

For the data exploration we use four basic views:

- 2D scatter plot,

- 3D scatter plot,

- histogram,

- parallel coordinates

Those views are well known and widely used. Although they are very general and simple, combined together they are powerful enough for exploring even very specific systems such as injection simulation [6].

A 2D scatter plot is used to show a relationship between two dimensions of the data set. Each record in the data set is represented with a point in the scatter plot. Additional information can be depicted using color coding or glyphs (instead of points). The 2D scatter plot used supports axis scaling and variable point size. Axis scaling allows for zooming to a part of the whole range. A scale slider positioned next to the axis depicts the current zoom position and ratio. The point size depends on the number of data set items falling into the same scatter plot point. Figure 5 illustrates the scale slider and the variable point size using a well-known iris data set [11]. The 3D scatter plot view is an extension of the 2D scatter plot into the third dimension.

A histogram displays a one-dimensional distribution. The $\mathrm{x}$-axis is divided into regular intervals and each histogram bin shows how

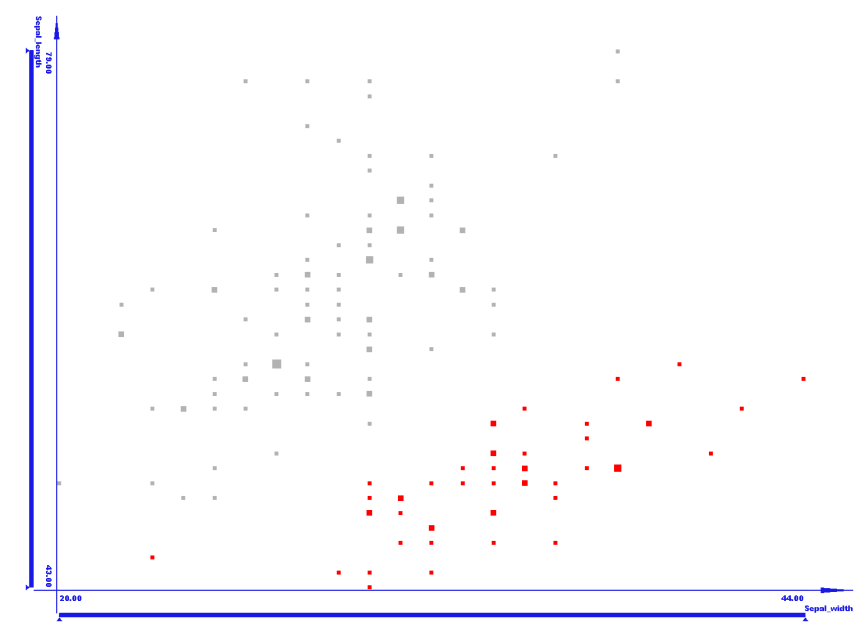

Figure 5: A simple scatter plot showing correlation between the petal width and height in the well known iris data set. Note the variable point size and the scale sliders.

many records have the particular dimension in the certain interval. Figure 6 shows an example histogram as we have used depicting petal length from the same iris data set [11]. The scale sliders can be used again to zoom in a particular area.

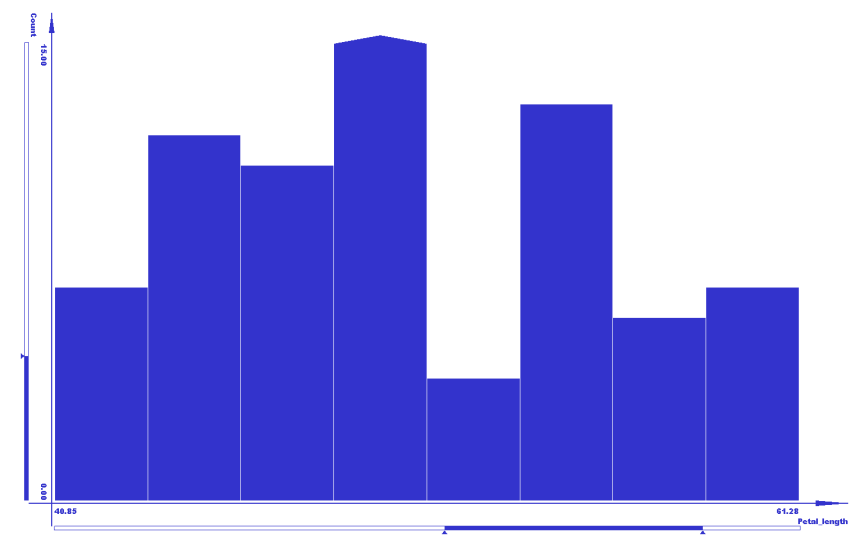

Figure 6: A basic histogram depicting a distribution of the petal length. Note a small triangle on the bin exceeding the maximum displayable $y$-axis value within the current range.

Parallel coordinates $[14,15]$ are often used to explore multidimensional data sets [13]. The main idea is to place more coordinate axes parallel to each other and to connect points representing values from a particular record on each axis with a line. In this way each record is represented with a poly line. Each axis can be zoomed independently, but the scale slider is shown for the highlighted axis only. Figure 7 illustrates the parallel coordinates view using the same data set.

\subsection{Linking and Brushing}

The views described so far do not significantly improve the analysis. The introduction of the interactive brushing and linking provides many advantages [7]. The idea behind brushing is that some ranges are marked (brushed) as interesting, and all data points within the brushed range are highlighted. The idea behind linking is to highlight the same items in all linked views [12]. All views 


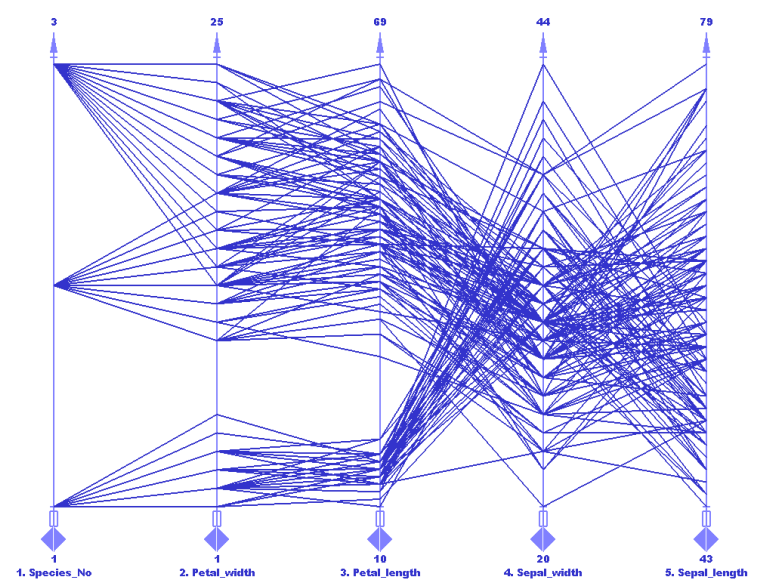

Figure 7: Parallel coordinates showing all 5 dimensions of the data set at once.

used in the analysis are linked and the user can interactively brush in each of the views.

The brush can also have a gradient color [16]. There is the start and the end color so that the actual color depends on the position of the item in the brushed range. The color from the original brushing area is propagated to all linked views. Figure 8 illustrates brushing and linking principles using the iris data set.

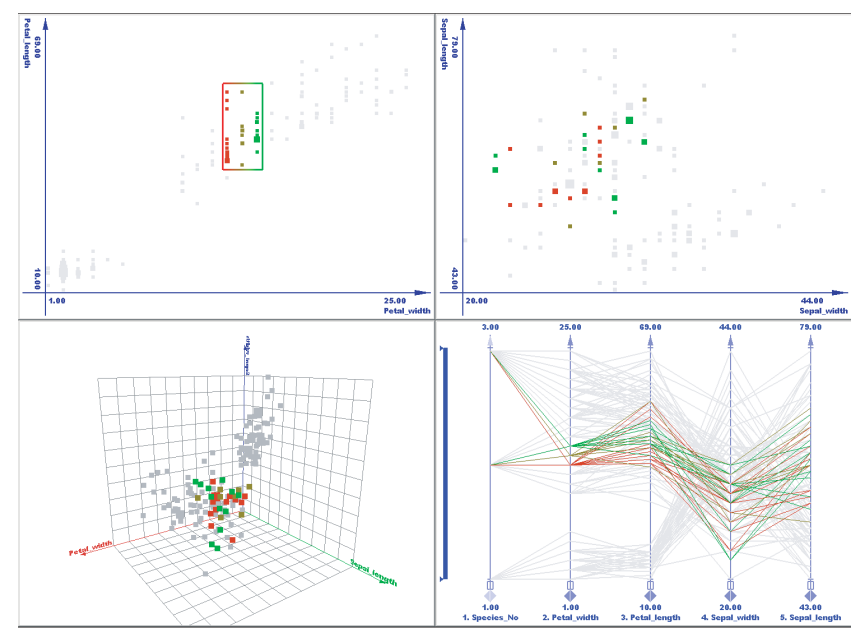

Figure 8: Linking and Brushing in multiple views. Note the color gradient on the brush. The brush color follows the petal width. The color coding in the linked views depicts the petal width.

\section{Analysis and Exploration of Injection Simula- TION}

Once the model of the injection system is created, there are many model parameters that can be varied. Those input parameters are called control parameters. The response parameters are those that change due to the changes in the control parameters. The main goal of the design process is to find a set of control parameters resulting in an optimum set of the response parameters. However, it is impossible to reach the optimum for all of the design goals (Section 2.1) so some compromises are necessary. The engineer has to decide where those compromises are made.
Table 1: The number of variations per control parameter

\begin{tabular}{cc} 
Parameter & number of variations \\
\hline R1 & 9 \\
R2 & 9 \\
R3 & 5 \\
$P_{\text {rail }}$ & 3 \\
$S V_{\text {open }}$ & 4 \\
$S V_{\text {close }}$ & 4
\end{tabular}

The model used for the case study has six control parameters, $R 1, R 2, R 3, P_{\text {rail }}, S V_{\text {open }}$, and $S V_{\text {close }}$ (Figure 3):

- $R 1$ is the area ratio of the inlet throttle $A_{\text {in }}$ to the outlet throttle $A_{\text {out }}$ of the control volume,

- $R 2$ is the area ratio of the control piston area $A_{c p}$ to the area of the bottom side of the nozzle needle $A_{n v}$,

- $R 3$ is the ratio of the inlet throttle area $A_{\text {in }}$ to the area of the control piston $A_{c p}$,

- $P_{\text {rail }}$ is the common rail pressure,

- $S V_{\text {open }}$ is the solenoid valve opening velocity, and

- $S V_{\text {close }}$ is the solenoid valve closing velocity.

There are six response parameters, $T_{\text {open }}, T_{\text {close }}, V_{\text {open }}, V_{\text {close }}$, $X_{\max }, m_{\text {inj }}$ :

- $T_{\text {open }}$ is the open response time for injection start,

- $T_{\text {close }}$ is the close response time for injection stop,

- $V_{\text {open }}$ is the needle opening velocity,

- $V_{\text {close }}$ is the needle closing velocity,

- $X_{\max }$ is the maximum needle lift, and

- $m_{i n j}$ is the injected fuel mass.

It is important to note that response parameters are scalars that are extracted from the time-dependent simulation results. For example, the needle-lift over time function is used to extract five of the response parameters (Figure 9). The sixth parameter, the injected fuel mass, is extracted from the injection rate over time.

Table 1 shows the number of variations for each control parameter. All possible combinations of the input parameters are used in simulations thus providing a total of 19440 items in the data set.

There are three main directions in the data exploration:

- finding invalid combinations of control parameters,

- finding combinations providing the desired results, and

- exploring tendencies. 


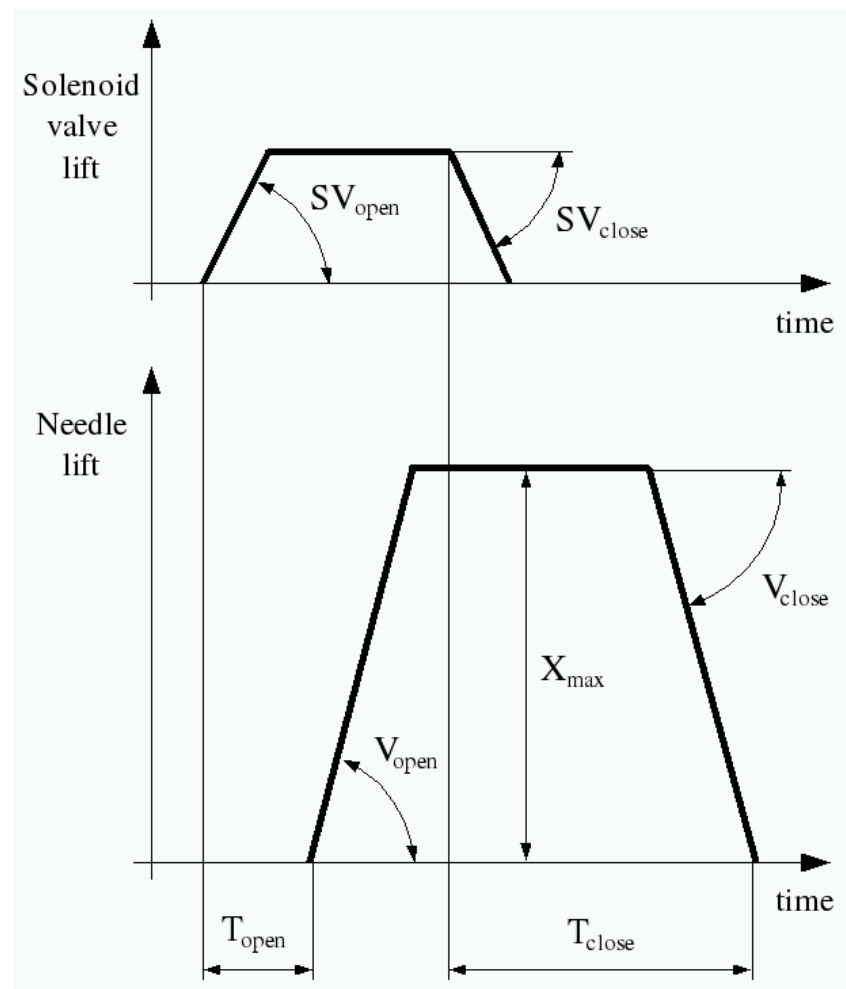

Figure 9: Characteristics of solenoid and valve lift

\subsection{Finding Invalid Combinations of Control Parameters}

Since the simulation was performed for every combination of the control parameters, there might be some combinations that result in an invalid operation of the injector. For example, when the needle lift is below a certain value(only interesting for pilot injection investigation, which is out of scope of this paper) injection will be insufficient (engine would not run). It is important to identify those combinations of control parameters and to exclude them from further analysis. Let us show how the interactive InfoVis techniques can be used to identify those cases. Since we are looking for a range in one control parameter, we use a histogram as a starting view. The histogram shows distribution of the maximum needle lift response parameter. We are interested in the bin corresponding to the lowest values of the maximum needle lift. If this bin is brushed, all related areas in the linked views are highlighted. We will first explore the influence of $R 1, R 2$, and $R 3$ control parameters on the maximum needle lift. The $3 \mathrm{D}$ scatter plot is used for this purpose. It is assumed that the low maximum needle lift results in the low injected fuel mass. Another histogram will be used to confirm this hypothesis.

Figure 10 shows this situation. In the upper left corner there is a histogram of the maximum needle lift. If we brush the first bin (small maximum needle lift values), the 3D scatter plot in the lower left corner of Figure 10 shows that brushed items have high $R 1$ ratio, high $R 2$ ratio, and whole range of $R 3$ ratios. Note that this does not necessarily mean that all items with high $R 1$ and high $R 2$ ratios have low maximum needle lift. If we want to exclude a subset of the control parameters from further analysis, we have to make sure that all items of the subset result in an invalid combination. To do this, we have included a $2 \mathrm{D}$ scatterplot showing $R 1$ and $R 2$, and brushed the upper right corner where both ratios are high. Figure 11 shows the result. The first bin highlighted in the injected fuel mass histogram shown in the upper right corner confirms the hypothesis.

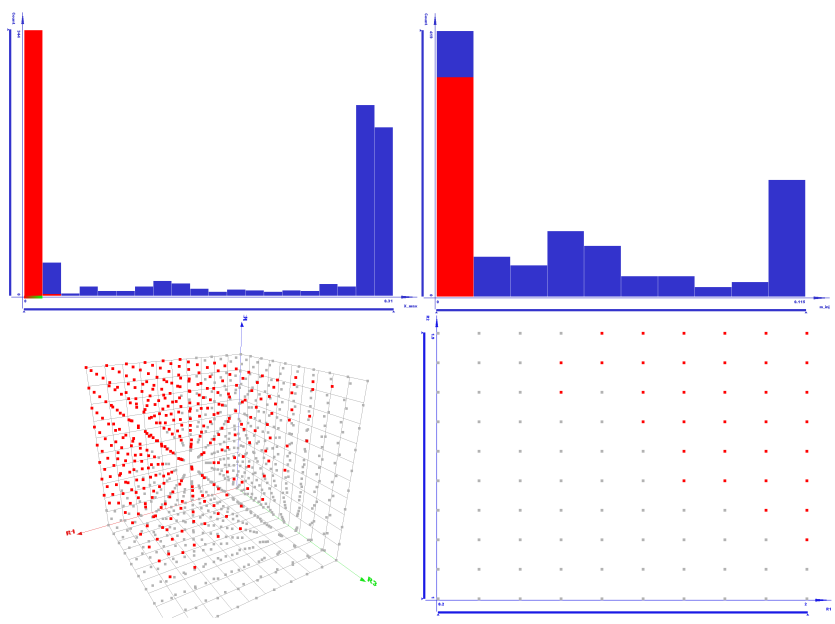

Figure 10: Interactive identification of invalid combinations. The low maximum needle lift items are brushed in the histogram (the upper left section). The 3D scatter plot in the lower left section shows brushed items in $R 1, R 2, R 3$ space. The $2 \mathrm{D}$ scatter plot in the lower right section is used for further brushing in order to find if the range of $R 1$ and $R 2$ can be excluded from further analysis. The histogram in the upper right section shows that all combinations with low maximum needle lift have low injected fuel mass, as expected.

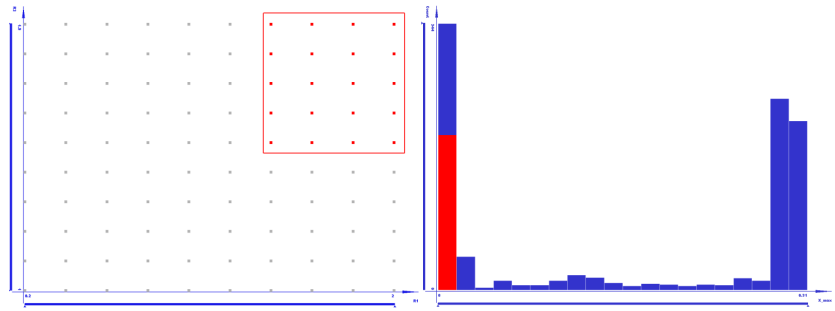

Figure 11: Brushing items with high $R 1$ and $R 2$ and showing that they can be excluded from further analysis.

There is a physical explanation for this case. High $R 1$ indicates the big inlet throttle area which results in almost no pressure drop in the control volume and almost no pressure difference between the control volume and the nozzle volume. That results in the opening force that is too weak.

High $R 2$ indicates a big control piston area that in effect results in a big closing force that acts together with needle spring force and closes the needle very hard.

\subsection{Finding Control Parameter Combinations Providing De- sired Results}

After the invalid cases have been excluded from the future analysis, engineers want to find out which input parameters result in a desired response parameters.

One of the very important issues when constructing injection systems is is to achieve very high needle closing velocities. This is important in order to prevent that the injected fuel is poorly prepared (because of the small injection pressure) or that the injection happens during the combustion process. Both would result in fuel not burning completely (or not at all). If such a fuel enters in the exhaust system, result is a high emission of environmentally unfriendly gases.

We will try to find the optimal designs by using InfoVis methods. Figure 12 in the upper left section shows the scatter plot of 

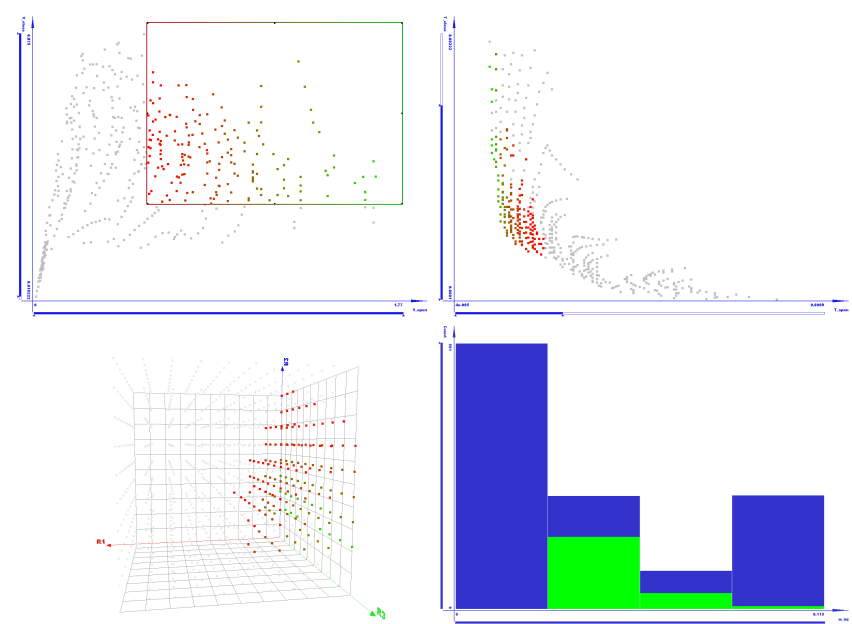

Figure 12: Finding out optimal combinations of control parameters. The 2D scatter plot in the upper left section shows opening and closing velocities. The desired range is brushed here. The 3D scatter plot in the lower left section shows brushed items in $R 1, R 2, R 3$ space. The 2D scatter plot in the upper right section shows opening and closing times. The histogram in the lower right section shows that most of the desired (brushed) combinations have satisfactory injected fuel mass.

opening and closing velocities. Note the scale slider on the closing velocity set to exclude very low closing velocities values. The exluded, low closing velocity cases, are actually also invalid combinations because the needle did not reach the closing position after the opening procedure is finished. The valve remains open. It is the opposite case to the invalid combination explored in the section 4.1, but the engine would not run either.

A desired case for engineers is when needle-opening and needleclosing velocities are large. This area is brushed in the scatter plot in the upper right section of the Figure 12. The 2D scatterplot in the upper right section shows the opening and closing response times. It is interesting (but not intuitive) that brushed data from the first scatterplot has short opening response time, but not always the short closing response time. Note that opening and closing times (Figure 9) do not directly influence opening and closing velocities. The $3 \mathrm{D}$ scatter plot in the lower-left section of Figure 12 shows that desired properties can be again achieved regardless of value of $R 3$, but only for some ranges of $R 1$ and $R 2$ control parameters. Finally, the histogram in the lower right section of Figure 12 shows that most of the brushed items have the satisfactory amount of injected fuel.

The interesting question here is why the points within the brushed area have the short open response time. The first guess by the engineers was that a larger outlet throttle enables faster propagation of pressure disturbance which was caused by the solenoid valve opening. This results in a faster flow through the control volume and bigger pressure drop. The engineers were curious if a bigger control piston area would damp this process. From the 3D scatter plot it may be seen that the optimum solution would be achieved for combinations with smaller $R 2$ parameter which implies that the control piston area must be smaller. It would generate bigger closing force which would increase the closing velocity and reduce the closing response time. In case of needle opening, it would result in a bigger reaction force.

In order to verify this hypothesis we have examined the data set again using parallel coordinates to simultaneously display all the affected parameters. Figure 13 illustrates the results. It can be seen that increasing of the $R 2$ parameter increases the open response time but at the same time decreases the close response time.

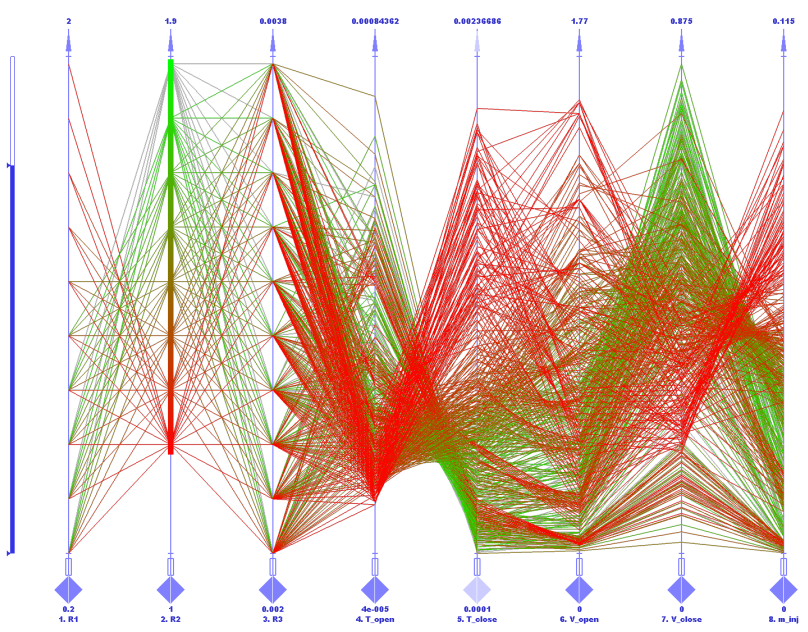

Figure 13: Parallel coordinates showing what is hapening if $R 2$ is increased.

\subsection{Exploring Tendencies}

In addition to finding invalid and optimum combinations it is also important to understand how the system works and how particular control parameters influence the system behaviour. This is especially true for novice engineers, or during the educational process. Interactive visual data exploration is an ideal tool for such tasks. The favourite interaction technique/tool is the moving of the existing brush across the view, and interactively observing linked views. Another convenient helper is the gradient color brush which is propagated to the linked views.

Figure 14 shows eight snapshots illustrating how an engineer used the system. Four different brush positions in are shown in the upper row. The idea is to change selected range of $R 1$ and $R 2$ control parameters in order to see how they influence the open response time and close response time (the lower row in Figure 14). Moving the brush from areas of big $R 2$ and small $R 1$ to areas of small $R 2$ shows that open response time is decreasing and close response time is increasing. If we want to find a physical explanation for such behavior, we have to focus on the force that acts on the needle. This force is a result of pressure differences between the control volume and the nozzle volume. For higher values of $R 2$ the closing force will be higher as a result of the bigger control piston area. To overcome this force and successfuly open the nozzle, a bigger pressure difference is needed and the process lasts longer. For a better value of close response time during the needle closing process, the higher values of $R 2$ help.

In another use case we illustrate the use of the single gradient color brush in order to explore the influence of $R 1$ parameter change on the injection process. The parallel coordinates view is used for this purpose (Figure 15).

The first axis represents the $R 1$ with almost entire range brushed using the gradient color brush. The second axis shows needle opening velocity. It can be noted that higher values of $R 1$ result in smaller needle opening velocities. The third axis depicts open response time. It behaves just the opposite compared to the needle opening velocity at second axis. An increase in $R 1$ value makes the open response time increase as well. The fourth axis shows the maximum needle lift. Note that a higher value of $R 1$ results in smaller maximum needle lift. The fifth axis shows the needle closing velocity. There is almost no correlation between $R 1$ and the needle closing velocity.

Higher $R 1$ values result in a smaller pressure drop between the common rail and the control volume. Pressure difference between 

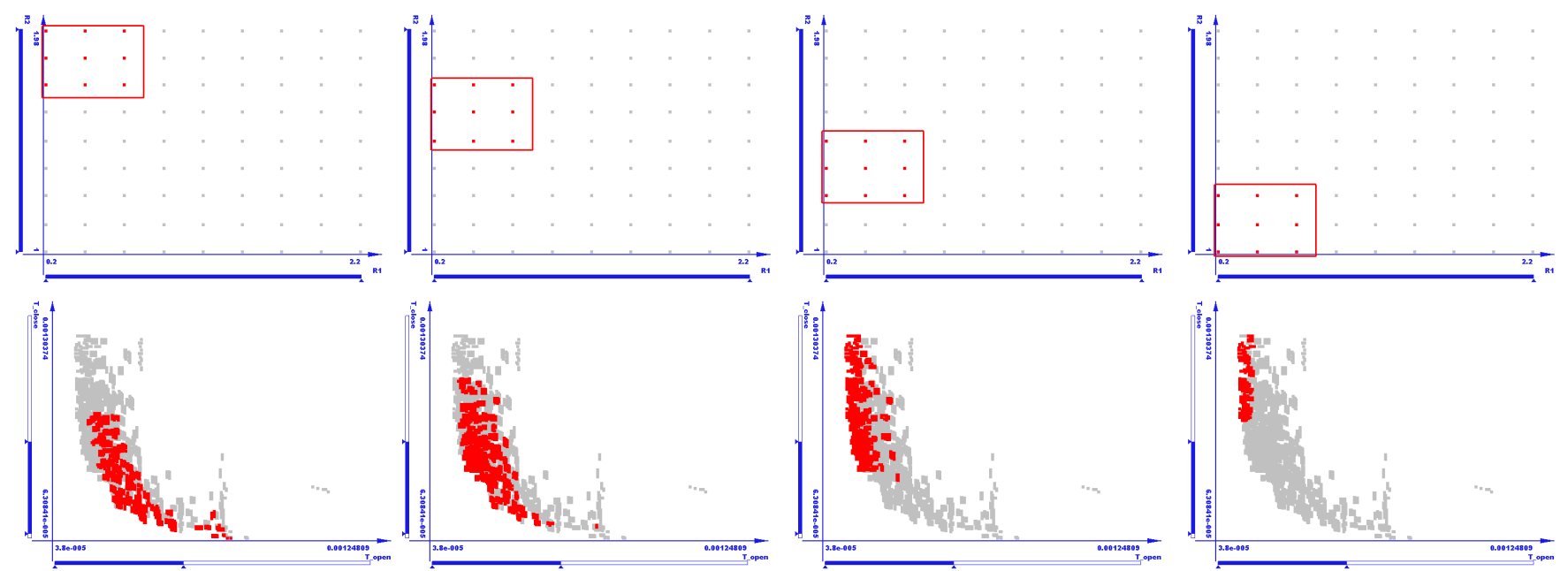

Figure 14: Exploring tendencies by moving the brush.

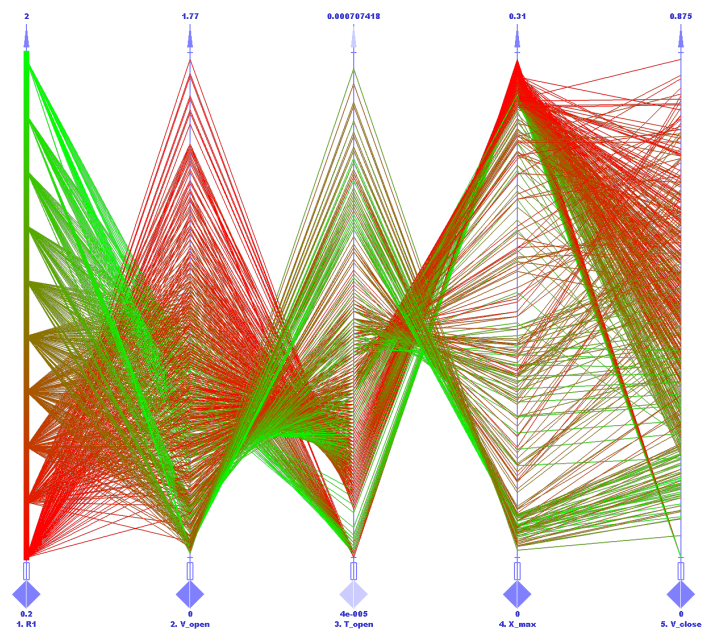

Figure 15: Exploring tendencies by using the single gradient color brush.

the control volume and the nozzle volume is smaller, which results in weaker opening force and smaller acceleration. Smaller opening force and acceleration produce smaller needle opening velocities. Because of the small pressure difference, the needle can not achieve high maximum lift. It is interesting that changes in $R 1$ do not have so transparent influence on the needle closing velocity.

An additional investigation (not presented here) showed that for the most of combinations with the higher needle closing velocity, the needle opening velocity stays small. The needle opening velocity is reduced in a number of combinations with fast opening and fast closing processes.

The third case illustrates the analysis of the influence of the common rail pressure on the injection system dynamics. The common rail pressure used in simulations was set to 1300 bar, 1500 bar and 1700 bar. We want to find out how the common rail pressure influences the values of response parameters that represent the system dynamics. Those parameters are: needle opening velocity, needle closing velocity, open response time, and close response time. We are interested in the possible $R 1$ and $R 2$ ranges, as well as in the injected fuel mass for each pressure value. Figure 16 shows the results. Increasing the common rail pressure provides wider range of

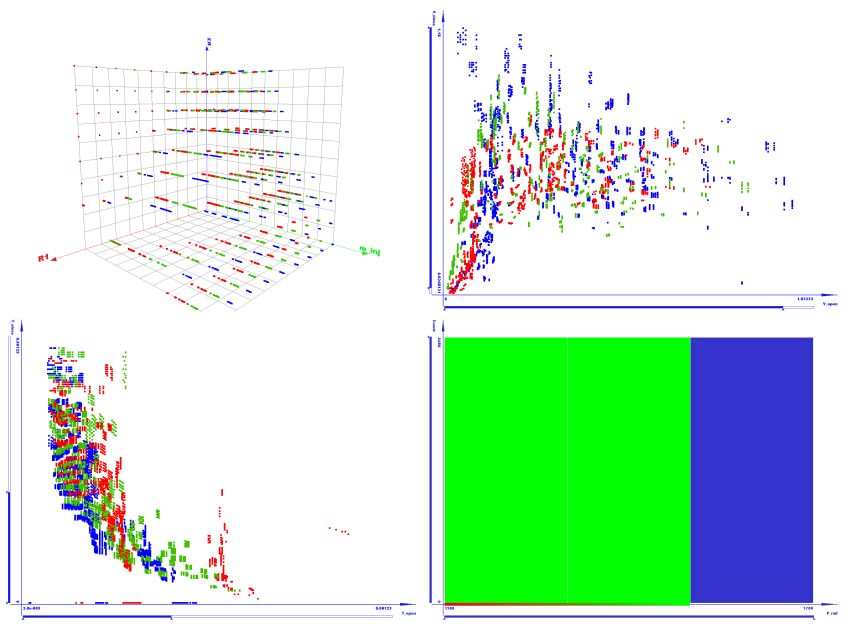

Figure 16: Sensitivity analysis: how the pressure influences the range of response parameters that represent the system dynamics.

possible solutions for system dynamics, which results in increased flexibility. On the other hand, the construction of a high pressure injection system is a complex and expensive task.

\section{Conclusion}

Conventional SciVis techniques may not be the most effective way of analysing the data in cases when there are many input and response parameters and the problem space dimensionality is reduced (e.g. 1D theory of fluid dynamics). In those situations, basic InfoVis techniques prove to be valuable in visualizing, understanding and analyzing the simulation data. In addition to basic InfoVis view types, linking and brushing techniques are used to connect different views of the parameters. The user can explore data in terms of analyzing different combinations of parameter values: finding invalid combinations of control parameters, finding combination of parameters providing desired results, and exploring tendencies. Such explorations are particularly well suited for parameterized simulations with huge amounts of result data.

We have used the ComVis tool to interactively visualize and explore injection simulation data set. Although the engineers who 
used the system had little previous experience with similar tools, they were very enthusiastic in using it. Previously used $2 \mathrm{D}$ charts and data tables are still indispensable, but it is much easier to understand the system behavior when interactive visualization is used. Although the commonly used numerical optimization methods are able to find the optimum set of control parameters, when the user wants to get a deeper insight in the system's behavior, the interactive visualization is much more efficient. Exploring the tendencies by using the interactive InfoVis system provides completely new qualities compared to a set of static $2 \mathrm{D}$ charts. When the number of parameters is large, the linked views and brushing prove to be the most intuitive and efficient way of exploring tendencies. This is particulary helpful for unexperienced engineers who need to learn about the influence of control parameters on the results of the simulated system, but also for experienced engineers who deal with the complex simulation models.

The case study of injection simulation demonstrates how the developed tool, ComVis, can provide a necessary functionality to explore data and establish physical interpretation of simulation results.

\section{ACKNOWLEDGMENTS}

We express special thanks to Helvig Hauser of VRVis for numerous discussions and help he provided. The data used is courtesy of AVL. Parts of this work have been carried out in the scope of applied and basic research at the VRVis Research Center which is funded by an Austrian governmental research program called K plus (www.kplus .at).

\section{REFERENCES}

[1] AVL List GmbH, http://www.avl.com

[2] B. Bederson and B. Shneiderman, editors. Readings in Information Visualization: using Vision to Think. Elsevier Science \& Technology Books, 1999.

[3] W. Boehner and K. Hummel. Common Rail Injection Systems for Commercial Diesel Vehicles. SAE Transactions, SAE 970345, 1997.

[4] S. K. Card, J. Mackinlay, and B. Shneiderman, editors. The Craft of Information Visualization: Reading and Reflections. Morgan Kaufmann Publishers, 2003.

[5] Combis, http://www.combis.hr.

[6] H. Doleisch. Visual Analysis of Complex Simulation Data using Multiple Heterogenous Views. $\mathrm{PhD}$ thesis, Vienna University of Technology, Austria, November 2004. See also http://www.VRVis .at/vis/resources/diss-HD/.

[7] H. Doleisch and H. Hauser. Smooth brushing for focus+context visualization of simulation data in 3D. Journal of WSCG, 10(1):147-154, 2002.

[8] H. Doleisch, M. Mayer, M. Gasser, P. Priesching, and H. Hauser. Interactive Feature Specifi cation for Simulation Data on Time-Varying Grids. In Conference on Simulation and Visualization 2005 (SimVis 2005), pages 291-304, 2005.

[9] H. Doleisch, M. Mayer, M. Gasser, R. Wanker, and H. Hauser. Case study: Visual analysis of complex, time-dependent simulation results of a diesel exhaust system. In Proc. of the 6th Joint IEEE TCVG - EG Symposium on Visualization (VisSym 2004), pages 91-96, Konstanz, Germany, May 2004

[10] S. P. Edwards, A. D. Pilley, S. Michon, and G. Fournier. The Optimisation of Common Rail FIE Equipped Engines Through the Use of Statistical Experimental Design, Mathematical Modeling and Genetic Algorithms . SAE Transactions, SAE 97346, 1997.

[11] R. A. Fisher. The use of multiple measurements in taxonomic problems. Ann. Eugenics, 7(1):179-188, 1936.

[12] D. Gresh, B. Rogowitz, R. Winslow, D. Scollan, and C. Yung. WEAVE: A system for visually linking 3D and statistical visualizations, applied to cardiac simulation and measurement data. In IEEE Visualization 2000, pages 489-492, 2000.
[13] A. Inselberg. Multidimensional detective. In INFOVIS '97: Proceedings of the 1997 IEEE Symposium on Information Visualization (InfoVis '97), page 100, Washington, DC, USA, 1997. IEEE Computer Society.

[14] A. Inselberg and B. Dimsdale. Parallel coordinates for visualizing multi-dimensional geometry. In CG International '87 on Computer graphics 1987, pages 25-44, New York, NY, USA, 1987. SpringerVerlag New York, Inc.

[15] Alfred Inselberg. A survey of parallel coordinates. In Hans-Christian Hege and Konrad Polthier, editors, Visualization and Mathematics, pages 167-179. Springer Verlag, Heidelberg, 1998.

[16] R. Kosara and Th. Rongitsch. Teaching old brushing new tricks. Technical Report TR-VRVis-2004-001, VRVis Research Center, 2004.

[17] R. S. Laramee, J. J. van Wijk, B. Jobard, and H. Hauser. ISA and IBFVS: Image Space Based Visualization of Flow on Surfaces. IEEE Transactions on Visualization and Computer Graphics, 10(6):637648, November 2004.

[18] E. Tufte. Envisioning Information. Graphics Press, Cheshire, CT 06410, 1990.

[19] E. Tufte. The Visual Display of Quantitative Information. Graphics Press, Cheshire, CT 06410, second edition, 2001.

[20] VRVis Research Center, http://www.vrvis.at. 